\title{
Comparison of BISAP score with Ranson's score in predicting Severe Acute Pancreatitis
}

Karki D, Tamang T, Maharjan D, Thapa P, Shrestha S

Kathmandu Medical College Teaching Hospital, Sinamangal, Kathmandu

Correspondence: Dimindra Karki, Resident, Department of Surgery, Kathmandu Medical College Teaching Hospital

Email: dimindra@gmail.com

\begin{abstract}
Objectives: To compare BISAP score with Ranson's scoring in predicting severity of acute pancreatitis
\end{abstract}

Methods: Extensive demographic, radiographic, and laboratory data from consecutive patients with AP admitted to our institution was collected between March 2014 to March 2015. Ranson's and BISAP score was calculated. Severity of pancreatitis was defined according to Atlanta classification. Sensitivity, Specificity, PPV, NPV of both the scoring system was calculated and compared.

Results: A total of 42 patients with diagnosis of acute pancreatitis were included during the study period. $21(50 \%)$ were male and $21(50 \%)$ were female. Mean age is $49.52 \pm 17.37$.Most common etiology was biliary (45\%) followed by alcohol (31\%). 20 (48\%) patients were categorized as severe pancreatitis according to Atlanta classification. 21 (50\%) patients had a Ranson's score of $\geq 3$ and 19 (45.24\%) patients had a BISAP score of 23. Both Ranson's and BISAP scoring system was statistically significant in determining SAP ( $p$-value $=0.002$ ). Sensitivity, specificity, PPV and NPV of Ranson's and BISAP score was calculated to be $75 \%, 72.72 \%, 71.43 \%$, $76.19 \%$ and $70 \%, 77.27 \%, 73.68 \%, 73.91 \%$. respectively. The AUC for SAP by Ranson's score is $0.7386 ; 95 \% \mathrm{Cl}$ ( $0.602-0.874)$ and BISAP score is $0.7364 ; 95 \% \mathrm{Cl}(0.599-0.872)$

\section{Conclusions:}

Both Ranson's and BISAP scoring system is similar in predicting SAP. However BISAP has the advantage due to its simplicity.

Key Words: Acute Pancreatitis, Severity, BISAP , Ranson's score 